\title{
A novel, data-driven heuristic framework for vessel weather routing
}

\author{
Christos Gkerekos ${ }^{\mathrm{a}, *}$, Iraklis Lazakis ${ }^{\mathrm{a}}$ \\ ${ }^{a}$ Dept. of Naval Architecture, Ocean and Marine Engineering, University of Strathclyde, \\ Glasgow/UK
}

\begin{abstract}
Fuel Oil Consumption (FOC) constitutes approximately 25\% of a vessel's operating costs and profoundly correlates with the adversity of the weather conditions along its route. Furthermore, increased FOC also leads to increased emissions. As shipping is turning page towards a greener, more sustainable future, it's crucial to leverage key insights from past routes in order to identify approaches that minimise both the financial cost of operations and their Green House Gas (GHG) footprint. This study presents a novel framework for vessel weather routing based on historical ship performance and current weather conditions at a discretised grid of points in conjunction with a data-driven model that can predict main engine FOC, Subsequently, a modified version of Dijkstra's algorithm that has been fitted with heuristics is applied recursively until an optimal route is obtained. The efficacy of the proposed framework is demonstrated through a case study concerning the optimal route selection for a 160,000 tonne DWT crude oil tanker sailing between the Gulf of Guinea and the Marseille anchorage.

Keywords: weather routing, fuel oil consumption prediction, ship energy efficiency, machine learning
\end{abstract}

\footnotetext{
${ }^{*}$ Corresponding author

Email address: christos.gkerekos@strath.ac.uk (Christos Gkerekos)
} 


\section{Introduction}

- Increasing a vessel's operational efficiency will yield a decrease in its Fuel Oil Consumption (FOC), positively impacting both its operating costs and its emissions footprint and is therefore pursued by a number of maritime industry 5 stakeholders such as ship operators, maritime regulators, and policy makers.

Sailing in adverse weather conditions can increase $\mathrm{FOC}$ and $\mathrm{CO}_{2}$ emissions by over 50\% (Prpić-Oršić et al. 2016). Furthermore, FOC constitutes approximately two-thirds of a vessel's voyage costs (Stopford, 2009). Therefore, identifying a ship route that avoids adverse weather can yield significant benefits in both financial and environmental terms. While weather routing is an active research topic, most studies apply first-principle approaches that do not accurately take into account a vessel's current condition and sailing peculiarities. Such peculiarities can include hull and engine degradation which can provide a more accurate way of suggesting the optimal route for a particular vessel.

The purpose of this paper is to present a novel framework for vessel weather routing based on historical ship performance and current weather conditions at a discretised grid of points. For this, a data-driven model that can predict main engine FOC is developed. Subsequently, a modified version of Dijkstra's algorithm that has been fitted with heuristics is applied recursively until an optimal route is obtained. This allows the creation of a vessel route that is derived based on the vessel's actual historical performance, increasing its robustness. The methodology developed in this paper can be used to produce more accurate and robust weather routes. This will help monitor and reduce vessels' FOC and $\mathrm{CO}_{2}$ emissions.

The remaining of this article is structured as follows. Section 2 refers to the research background on weather routing, including an overview of previous attempts at modelling $\mathrm{FOC}$. Section 3 elaborates on the proposed methodology, focusing on aspects of data pre-processing, model training and verification. Section 4 details the setup of the case studies for the verification of the proposed work, presenting and discussing the results. Finally, in Section 5 , overall 
conclusions are provided.

\section{Literature review}

This section provides an overview of scientific literature, pertinent to this study. First, methodologies relevant to FOC modelling are analysed. Following this, an overview of weather routing approaches are discussed. A synopsis of data-driven techniques relevant to the modelling requirements of this paper are then presented.

Beşikçi et al. (2016) compare shallow Artificial Neural Network (ANN) and Multiple Regression (MR) models for the prediction of ship FOC at various

40 operational conditions. The ANN model was shown to have increased accuracy compared to the MR model. The output of this ANN model is proposed to be used as the backbone of a Decision Support System (DSS) functionality, offering a number of optimised sailing parameters for reduced FOC and environmental jimpact. Contrary to that, Wang et al. (2018) propose a Least Absolute Shrink-

45 age and Selection Operator (LASSO) regression model for the estimation of a vessel's FOC. The authors note that this model has an optimal performance

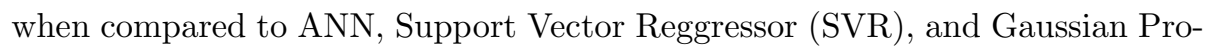
cesses (GPs) models in a case study utilising low-frequency data obtained from a fleet of containerships. Petersen et al. (2012) also investigate ANN models for ferry main engine $\mathrm{FOC}$ modelling. The output of the model produced was also used as the backbone of a trim optimisation DSS, Coraddu et al. (2017) perform a comparison of white, grey, and black box models for the FOC forecasting of a Handymax chemical/product tanker, noting that grey-box models can effectively forecast $\mathrm{FOC}$ in the presence of only limited historical data. Gkerekos

55 et al. $(2018,2019)$ conducted a thorough examination of data-driven modelling approaches to identify the efficacy of different models in modelling vessel $\mathrm{FOC}$ consumption based on different sailing data sources.

Armstrong and Banks (2015) provide an integrated overview of energy efficiency improvements for vessel sailing, highlighting the necessity of a set of well- 
defined Key Performance Indicators (KPIs) to simplify performance tracking. Acomi and Acomi (2014) suggest the use of EEOI as a KPI for the amelioration of vessel sailing FOC efficiency.

Walther et al. (2016) provide an overview of state-of-the-art approaches for weather routing, noting that all approaches present significant benefits and deficiencies and the weather routing topic remains heavily researched. Veneti et al. (2017) suggest a detailed framework for weather routing based on a shortestpath algorithm, where vessel's FOC is a static approximation based on first principles. Accordingly, Vettor and Guedes Soares (2016) combine a first-principle ship response estimation with a genetic algorithm approach for the derivation of an optimal vessel route. Lin et al. (2013) propose a combination of a mathematical model that predicts ship performance with a three-dimensional modified isochrone (3DMI) method for weather routing. Similarly, Roh (2013) suggests a framework for weather routing based on first principles resistance-based FOC estimation and the isochrone method for weather routing. Lu et al. (2015) propose 75 another semi-empirical method for the prediction of operational performance of ships based on modelling ship' resistance. The ship's operational performance is then modelled, taking into consideration the weather and relevant sea state and utilised to optimise the ship's route.

From the above, it can be deduced that weather routing is an active research so field with multiple different approaches being realised concurrently. However, up to present, most studies focus on first-principle methods for the estimation of a vessel's performance. Due to this, the approaches suggested do not accurately take into account a vessel's current condition and sailing peculiarities. This work aims to alleviate this gap by proposing a framework that includes data preprocessing, data-driven modelling that takes into account a vessel's particular characteristics and current performance and provides an optimised route based on those parameters and the forecasted sea conditions. 


\section{Methodology}

The methodology elaborated in this section consists of the following steps: a)

90 derivation of an accurate FOC model; d) the design of a weather routing module that consider several trial routes to offer decision support on optimal routing; and e) the derivation of number of KPIs pertinent to vessel performance.

A visual representation of the developed methodology is presented in Figure 95 1. illustrating these steps and their interconnections.

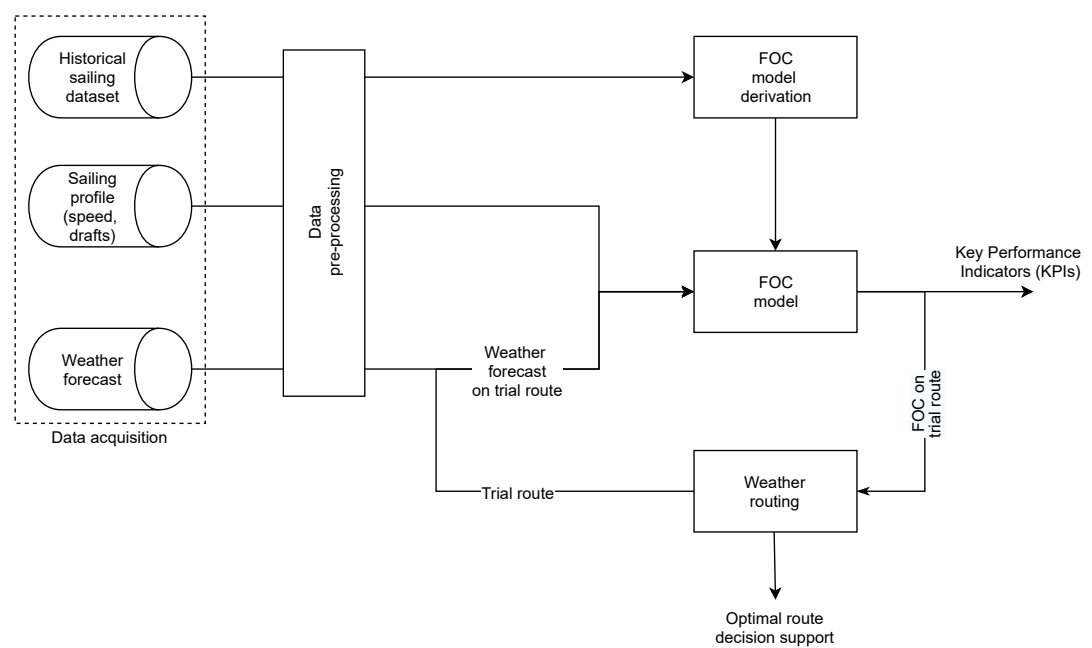

Figure 1: Visual representation of the suggested methodology.

\subsection{Data acquisition}

The Data Acquisition (DAQ) required for model training can be obtained either through an Automated Data Logging \& Monitoring (ADLM) system or through noon-reports. Previous research has shown that ADLM systems can increase modelling accuracy by $5-7 \%$ and reduce the required data acquisition period by up to $90 \%$ (Gkerekos et al. 2019). However, ADLM systems are not always available and in those cases noon-reports are used instead.

For weather routing, a second dataset containing ocean analysis and forecast data for the sailing region is required. This is obtained through weather 
providers such as Copernicus Marine Environment Monitoring Service (CMEMS) and depending on provider and attribute characteristics come with different spatial and temporal resolutions. For example, CMEMS global wave forecast has a maximum spatial resolution of 1/12 degree and a 3-hourly temporal resolution, providing 5 days of forecast.

110

Due to the existence of potential measuring anomalies, lack of data, and undesirable data points (e.g. engine transients), pre-processing follows the data acquisition phase for both datasets.

\subsection{Data pre-processing}

In the case of data required for model training, engine transients and recording anomalies are identified and rejected. Regarding the weather forecast dataset, instances with missing information are imputed to obtain an uninterrupted dataset.

\subsubsection{Engine transients rejection}

The derived FOC model aims to provide a prediction of the vessel's FOC at discretised segments that, depending on the spatial resolution of the underlying weather forecast, often span several hours. Based on that, only steady-state (i.e. continuous) historical sailing data are used for its training.

口 In this respect, Main Engine (M/E) Original Equipment Manufacturers (OEMs) provide a minimum engine speed for continuous operation (MAN B\&W

125 Diesel A/S, 2004), usually at $15-25 \%$ of the engine's nominal maximum continuous $\left(L_{1}\right)$ speed. Any observations corresponding to measured speed below that threshold are then rejected as an engine transient or manoeuvring. Additionally, observations where the engine power varies by more than $5 \%$ hourly are also discarded as the FOC is only trained on steady-state data due to the low temporal resolution of the weather forecast provider (Tsitsilonis and Theotokatos, 2018). 


\subsubsection{Recording anomalies rejection}

Sailing data acquisition may potentially introduce inconsistent and/or faulty data entries (e.g. due to sensor anomalies or human error) that need to be identified and rejected at this stage. Applying the Central Limit Theorem (CLT) given that sensor data are aggregated every few seconds or minutes, data can be assumed to follow a normal distribution. Therefore, $99.7 \%$ of normal data should be within $\mu \pm 3 \sigma$, where $\mu$ corresponds to the mean value of each attribute and $\sigma$ to its standard deviation. Hence, observations outwith this range can be rejected as anomalous without affecting the vast majority of normal points (Gkerekos et al., 2019).

\subsubsection{Weather forecast imputation}

n To impute missing weather forecast data point with missing attributes, Multiple Imputation by Chained Equations (MICE) algorithm is applied. MICE

145 algorithm is an imputation approach that fits a number of regression models to the dataset in order to infer missing values (Shah et al., 2014).

A brief description of the steps required for the application of MICE algorithm are as follows (Cheliotis et al., 2019):

1. Replace all missing values with the mean value of their attribute as an initialisation step. Keep track of instances where this has happened.

2. Revert the missing values of a single attribute to missing.

3. Fit a linear regression model to predict missing values of that attribute based on some (or all) other attributes.

4. Repeat steps 2 and 3 until no attributes have missing points.

5. Repeat steps $1-4$ for a predetermined number of cycles.

\subsubsection{Feature engineering}

All ocean analysis and forecast data follow a space-fixed frame of reference, i.e. are either provided in the form of a northward and eastward component or 
in that of an angle and a magnitude. As the effect of the weather elements

depends on the current course of the ship, these measurements are transformed into a body-fixed frame of reference.

To transform measurements that are provided in a northward $(n w)$ and eastward (ew) component into the vessel body-fixed frame of reference sailing at a bearing $b r$ and obtain a longitudinal $(l g)$ and transverse $(t r)$ component, the following formulas can be used:

$$
\begin{aligned}
& l g=n w \cos (b r)+e w \sin (b r) \\
& t r=|e w \cos (b r)|+n w \sin (b r)
\end{aligned}
$$

In the case where the measurements are described by a magnitude $M$ and an angle $\alpha$, they can be transformed into the vessel body-fixed frame of reference sailing at a bearing $b r$ and obtain longitudinal $(l g)$ and transverse $(t r)$ components by applying the following formulas:

$$
\begin{aligned}
& l g=M \cos (\alpha-b r) \\
& t r=|M \sin (\alpha-b r)|
\end{aligned}
$$

\subsection{FOC model derivation}

A thorough study of the FOC modelling problem (Gkerekos et al. 2019) has identified that Extra Trees Regressors (ETRs), Random Forest Regressors (RFRs), SVRs, and ANNs yielded the best performance results. Out of those, only ANNS and SVRs offer the required generalisation capabilities required for weather routing. Comparing ANNs and SVRs, ANNs yielded better results when fed with ADLM-provided data. Therefore, in this work a multi-layer (i.e., deep) ANN will be developed for FOC modelling.

\subsubsection{Artificial Neural Networks}

ANNS are an interconnected assembly of simple processing elements, units or nodes, whose functionality is loosely based on the animal neuron (Gurney, 1997). Nodes connect to each other based on inter-unit connection strengths 
called weights. ANNS can adopt different architectures that enhance their performance in machine learning tasks, including classification and regression. Due to this, they are extremely versatile and can accurately model complex nonlinear behaviours.

Tunable elements of a model can be grouped in two generalised categories, parameters and hyperparameters. Whereas parameters are elements of the model that are directly learnt from the training data, hyperparameters cannot be directly estimated from the training data as there is no analytical formula available to calculate an appropriate value (Kuhn and Johnson, 2013). Therefore, hyperparameters are specified manually, often following heuristics. The main hyperparameters that affect the performance of an ANN are the number of hidden (i.e., between input and output) layers, the number of nodes per layer, the activation function at each layer, the batch size, and in the case of deep ANNS the dropout rate.

The number of layers controls the depth of the ANN whilst the number of nodes per layer controls its width. Increasing the width of a layer increases its memorisation capabilities whereas increasing the depth of a network increases its capability of learning features at different levels of abstraction. In both cases, a more-is-better approach is not sensible as it would cause the model to overfit the training dataset.

Given an ANN regressor with an input layer $\mathbf{x}$, a hidden layer with $M$ nodes $Z_{m}$, and an output layer consisting of a single node, each node is of the form (Hastie et al., 2009)

$$
\begin{aligned}
Z_{m} & =\sigma\left(\alpha_{0 m}+a_{m}^{T} \mathbf{x}\right) \\
Y & =f(\mathbf{x})=g\left(w_{0}+w^{T} Z\right)
\end{aligned}
$$

where $Z=\left(Z_{1}, Z_{2}, \ldots Z_{M}\right), \sigma(\cdot)$ is the activation function and $g(\cdot)$ is the userselectable output function. In the case of regression, the identity function is used as output function (Hastie et al. 2009). Currently tanh or ReLu (Rectified Linear Units) are the preferred activation function choices. The formulation presented above can easily be extended for the case of multiple hidden layers 
by using the output of each layer as input for the next, and so forth.

Dropout is applied in deep ANNs to reduce overfitting of models by randomly dropping nodes (and their connections) from the ANN during training (Srivastava et al., 2014). Dropout rate controls the probability of this random effect happening at each node. An ANN with and without dropout is depicted in Figure 2 ,

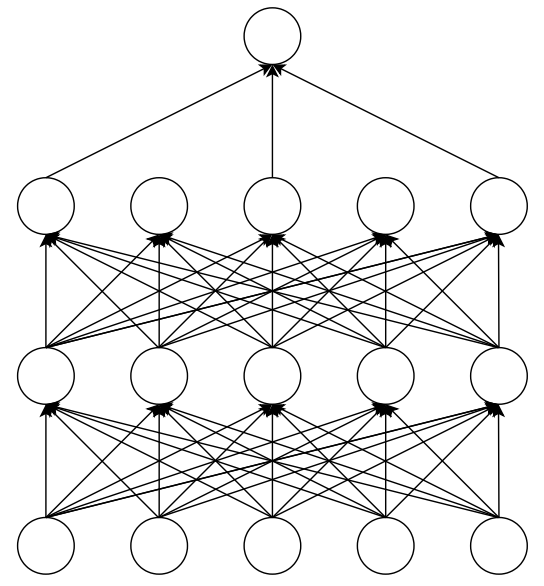

(a) Standard Neural Network

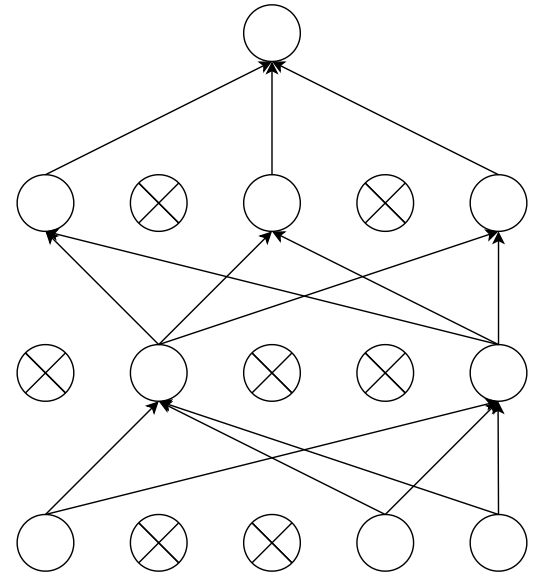

(b) After applying dropout

Figure 2: Example of a 2-layer ANN before (a) and after (b) the application of Dropout. Crossed units have been dropped and the dropout rate controls the probability of each unit dropping. Adapted from (Srivastava et al. 2014).

Batch size defines the number of training examples fed to the model before updating its parameters. A larger batch size will require a reduced computational cost but may converge to local minima instead of global Goodfellow et al. 2016).

\subsubsection{FOC model derivation \&3 hyperparameters' search space}

The overall architecture of the developed ANN is depicted in Figure 3 and the search space of the model's hyperparameters in Table 1 . The existence of hidden layers 3 and 4 depends on the selected value of network depth. Accordingly, the width, dropout rate, and activation function are considered individually for each layer. 


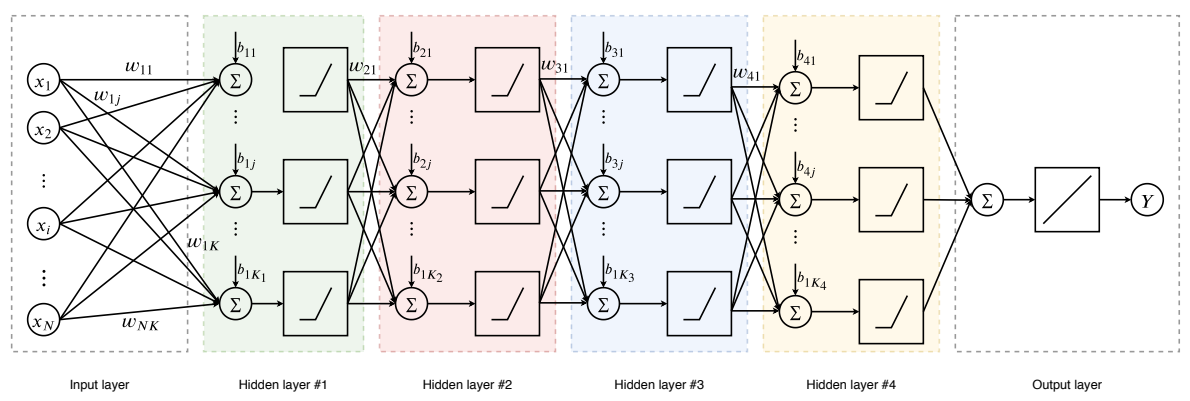

Figure 3: Visual representation of the developed ANN

Table 1: Search space for ANN hyperparameters

\begin{tabular}{ll} 
Hyperparameter & Search space \\
\hline Network depth & $\{2,3,4\}$ \\
Layer width & $\{8,16,32,64,128,256,512\}$ \\
Dropout rate & {$[0,1)$} \\
Activation function & $\{$ ReLU, Sigmoid $\}$ \\
Batch size & $\{32,64,128\}$
\end{tabular}

Due to the high computational cost of testing every possible combination of hyperparameters to identify the optimal, an optimisation algorithm is used. In this case, a Tree-structured Parzen Estimator Approach (TPE) is applied (Bergstra et al., 2011).

Any number of available measurements $x_{i}$ are used as model input and the model's output is the FOC for that route. It should be noted at this point that the same input measurements used at training must also be available at execution time for the model to work as expected.

\subsection{Weather routing}

A modified version of Dijkstra's algorithm that has been fitted with heuristics 225 is applied recursively to obtain an optimal weather route.

Dijkstra's algorithm (Dijkstra, 1959) is an algorithm for identifying the shortest paths between nodes in a static graph. Dijkstra's algorithm in pseu- 
docode form is presented in Algorithm 1 .

However, weather routing is a shortest path problem on a dynamic graph as the cost of moving between nodes depends on the present weather conditions that vary as a function of time. For this reason, the following steps are followed to use Dijkstra's algorithm on a dynamic graph for weather routing:

1. Looping through all graph vertices $v$, apply Dijkstra's algorithm on the graph to identify the shortest path connecting source and target vertices through $v_{i}$. It should be noted that at this point, only the shortest distance is considered and not the vessel's FOC over the identified paths.

2. Calculate the vessel's FOC over the shortest paths, retain a user-selectable number of vertices $v_{\text {opt }}$ that yield paths with lowest FOC.

3. Looping through $v_{\text {opt }}$ and $v$, apply Dijkstra's algorithm on the graph to identify the shortest path connecting source and target vertices through $v_{o p t_{i}}$ and $v_{i}$.

4. Calculate the vessel's FOC over the shortest paths, retain a user-selectable number of vertice tuples $v_{\text {opt }}$ that yield paths with lowest FOC.

5. Repeat steps $3-4$ until no change in $v_{o p t}$ is observed.

This is also presented in pseudocode form in Algorithm 2

\subsection{Key Performance Indicators}

A number of KPIS pertinent to vessel performance, efficiency and condition are presented below, along with their mathematical formulation and application practicalities.

250

As the KPIs discussed below are based on the FOC modelling framework developed in Section 3.3, a well-validated model is considered a prerequisite. Naturally, these KPIs are calculated at different points, across multiple routes and then averaged out over time-windows to create trendline plots of their deviation. 


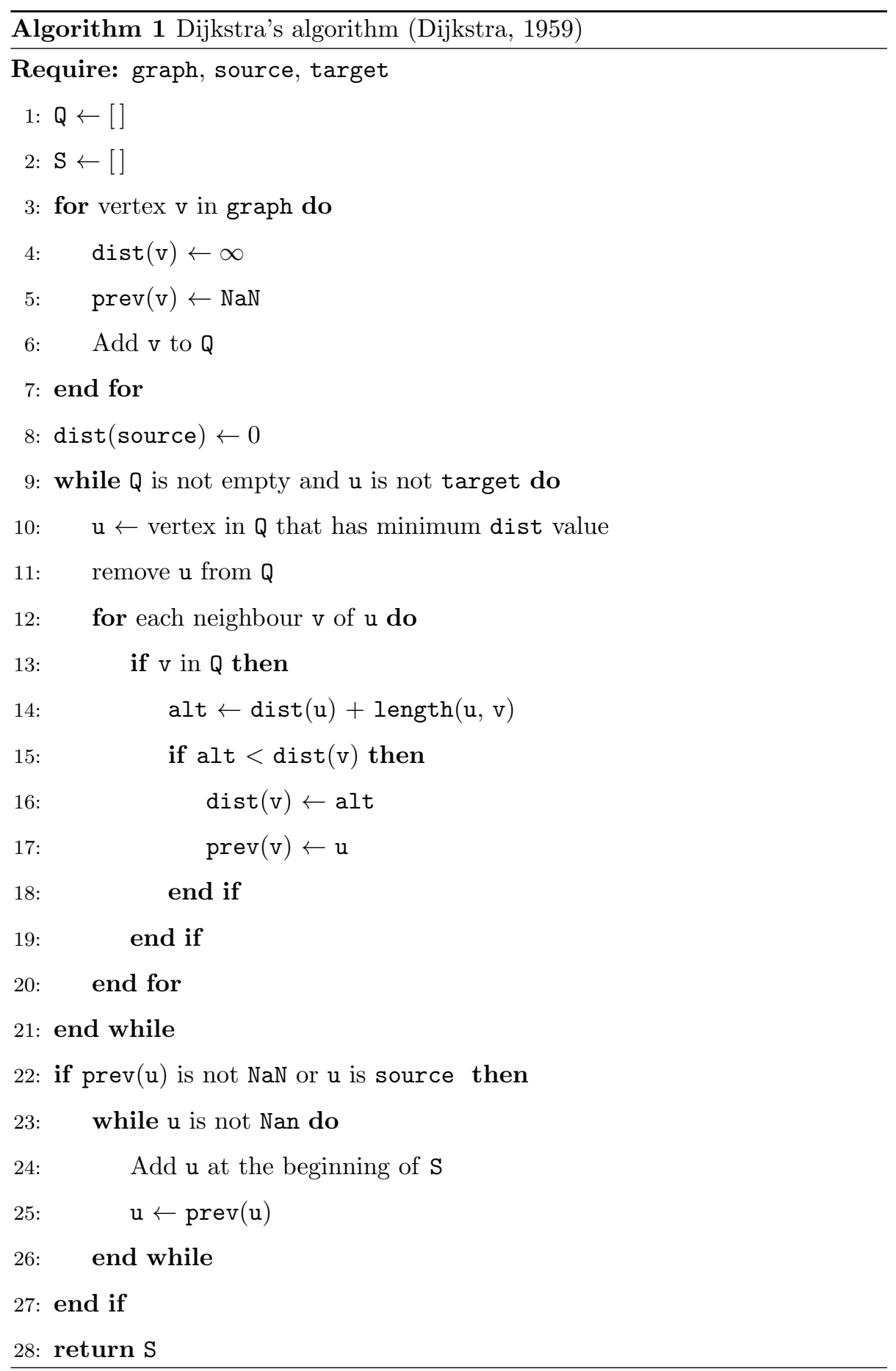




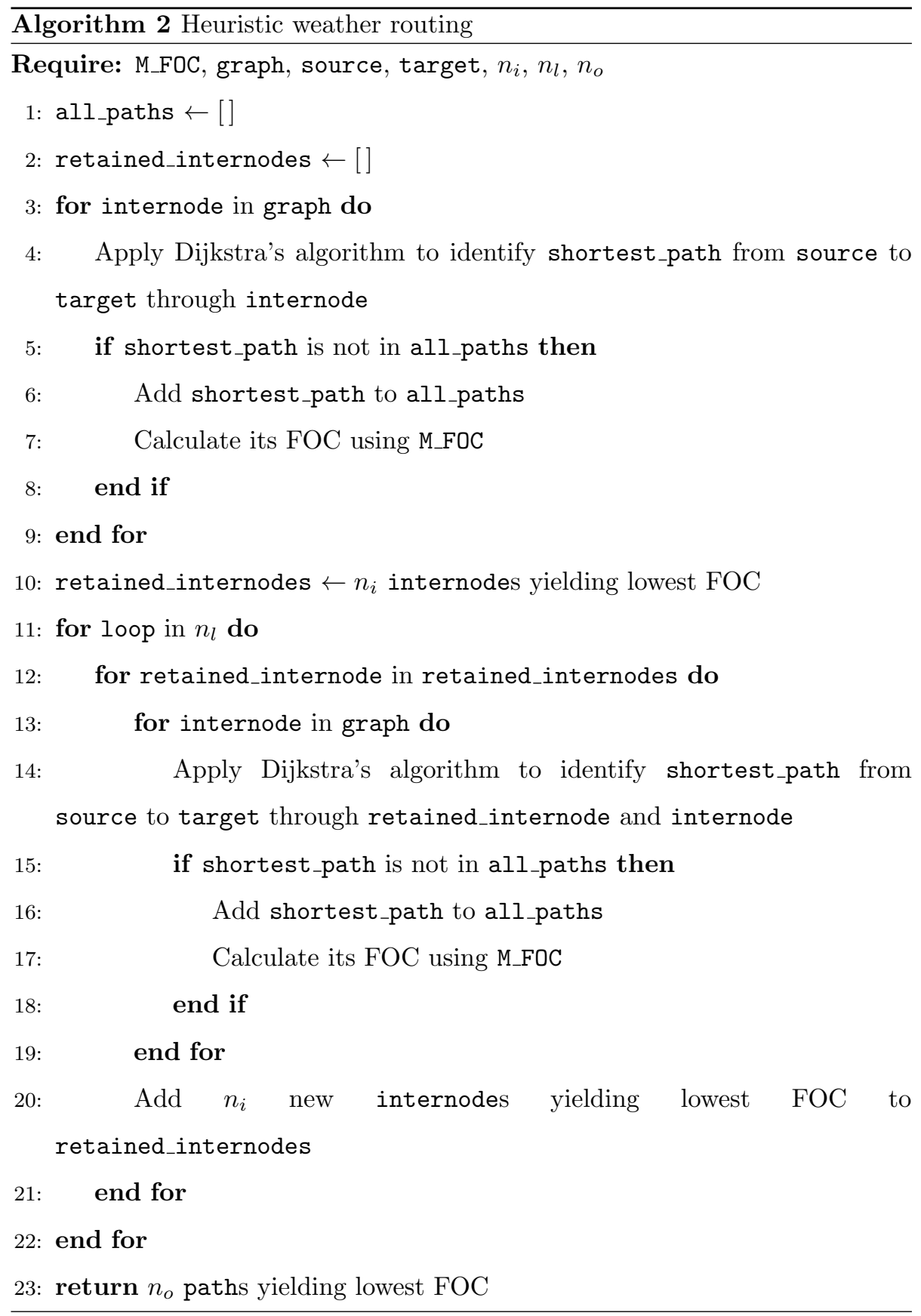


It should be noted that ideally, a separate model should be created for KPI purposes following the process described in Section 3.3 while utilising data of acceptable performance, e.g. data obtained following a hull cleaning or major overhaul. This is due to an inherent trade-off between the Route Optimisation module requiring a $\mathrm{FOC}$ model that accurately predict a vessel's behaviour at its current state and the KPIs requiring a model that always reflects a vessel's optimal behaviour as a condition/performance baseline.

\subsubsection{KPI I: Vessel Sailing Performance}

The ratio between measured and expected distance travelled over any amount of fuel consumed can be used to quantify the sailing performance of a vessel.

Mathematically, this can be formulated as

$$
K P I_{I}=\frac{d_{m f}-d_{e f}}{d_{e f}}
$$

\subsubsection{KPI III: Vessel Condition}

To monitor the condition of the coupled hull and propeller system in the form of a KPI the required power can be compared to the model's estimation. Therefore, this KPI can be mathematically formulated as

$$
K P I_{I I I}=\frac{P_{m}-P_{e}}{P_{e}}
$$


where $P_{m}$ refers to the measured propulsion power for any sailing conditions and $P_{e}$ to the model's estimation.

\subsubsection{KPI IV: M/E Performance \&3 Condition}

In order to monitor the M/E condition and performance, a KPI based on the comparison between the measured and recorded Specific Fuel Oil Consumption (SFOC) values. This KPI can be mathematically formulated as

$$
K P I_{I V}=\frac{S F O C_{m}-S F O C_{e}}{S F O C_{e}}
$$

where $S F O C_{m}$ refers to the measured $S \mathrm{SFOC}$ for any sailing conditions and $S F O C_{e}$ to the model's estimation.

\section{Methodology Application \& Results}

Having elaborated the framework components in Section 3, the framework is applied to a case study utilising actual ship raw data for verification and validation purposes. This case study concerns a 160,000 tonne DWT crude oil tanker sailing between the Gulf of Guinea and the Marseille anchorage, and can be extended as-is to any other ship type and voyage. This above route was selected as it represented a typical voyage of the aforementioned vessel for which data were available at the time of the preparation of this paper.

Data pertaining to 10 ship voyages were provided for FOC model training and testing. In total, 2713 data points were obtained after merging all provided information. 647 were dropped after filtering for anomalies and transients, leaving 2066 points in the dataset. The parameters obtained are presented in Table 2 .

The ocean analysis \& forecast data used for weather routing contains measurements $7-14$ of Table 2 at 368 ocean locations corresponding to a graph the encompasses the source and target navigation points at a resolution of $1^{\circ}$ in both latitude and longitude at a time frequency of 3 hours. Intermediate points are interpolated in space and time. 


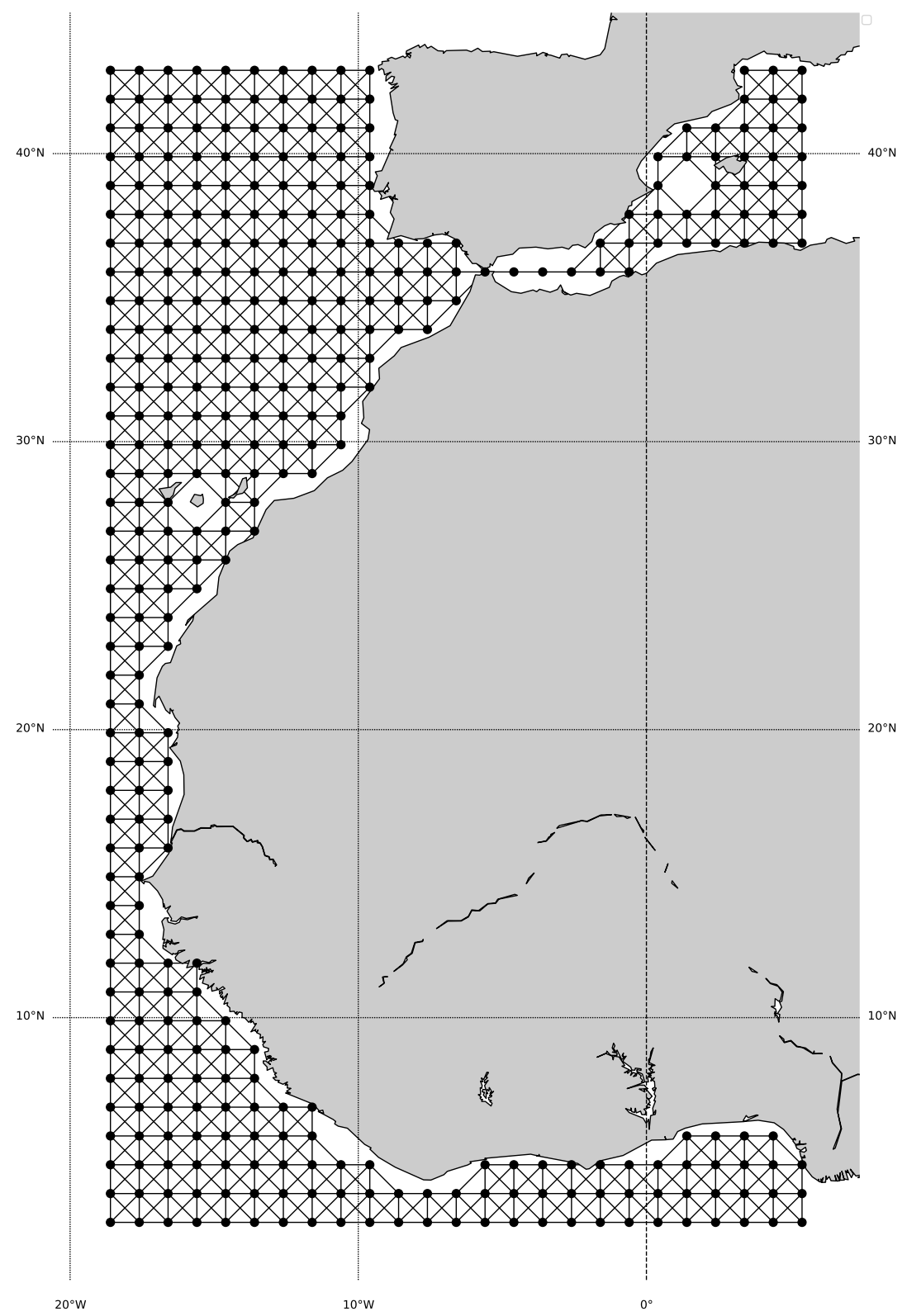

Figure 4: Visual representation of graph points where weather information was available. 
Table 2: Overview of vessel sailing dataset used for FOC model training.

\begin{tabular}{rlc}
\hline$\#$ & Name & Units \\
\hline 1 & Draft fore & $\mathrm{m}$ \\
2 & Draft aft & $\mathrm{m}$ \\
3 & Engine speed & $\mathrm{rev} / \mathrm{min}$ \\
4 & Engine FOC & $\mathrm{kg} / \mathrm{hr}$ \\
5 & Engine power & $\mathrm{kW}$ \\
6 & Vessel over ground speed & $\mathrm{knots}$ \\
7 & Eastward current & $\mathrm{knots}$ \\
8 & Northward current & $\mathrm{knots}$ \\
9 & Primary swell wave significant height & $\mathrm{m}$ \\
10 & Secondary swell wave significant height & $\mathrm{m}$ \\
11 & Primary swell wave direction & degrees \\
12 & Secondary swell wave direction & degrees \\
13 & Wave significant height & $\mathrm{m}$ \\
14 & Wave direction & degrees \\
\hline
\end{tabular}

\subsection{FOC model derivation}

Applying the FOC modelling framework discussed in Section 3.3, the optimal ANN hyperparameters were identified as shown in Table 3

Testing the model derived on a test set that corresponds to real vessel sailing data, an $R^{2}$ of $89.4 \%$ was obtained. The results obtained are presented in the form of a Y-Y plot in Figure 5. Furthermore, the error between predicted and actual $\mathrm{FOC}$ for the test set is presented in time-series form in Figure 6. In this graph, $95.5 \%$ of predicted points are shown to be within $10 \%$ of their actual values. A limited number of outliers cross this value and present increased error values but these values are only obtained sporadically and can be attributed to discrepancies between the actual weather conditions observed in the vessel's ambient space and the weather provider's extrapolated data. In practical terms, 
Table 3: Identified optimal values for ANN hyperparameters

\begin{tabular}{ll} 
Hyperparameter & Value \\
\hline Network depth & 4 \\
Layer 1 width & 256 \\
Layer 2 width & 256 \\
Layer 3 width & 64 \\
Layer 4 width & 512 \\
Layer 1 Dropout rate & 0.36 \\
Layer 2 Dropout rate & 0.47 \\
Layer 3 Dropout rate & 0.04 \\
Layer 4 Dropout rate & 0.53 \\
Layer 1 Activation function & ReLU \\
Layer 2 Activation function & ReLU \\
Layer 3 Activation function & Sigmoid \\
Layer 4 Activation function & ReLU \\
Batch size & 32
\end{tabular}


having a model that can achieve a high $R^{2}$ on real vessel sailing data is a practical validation of the model's fitness for this purpose.

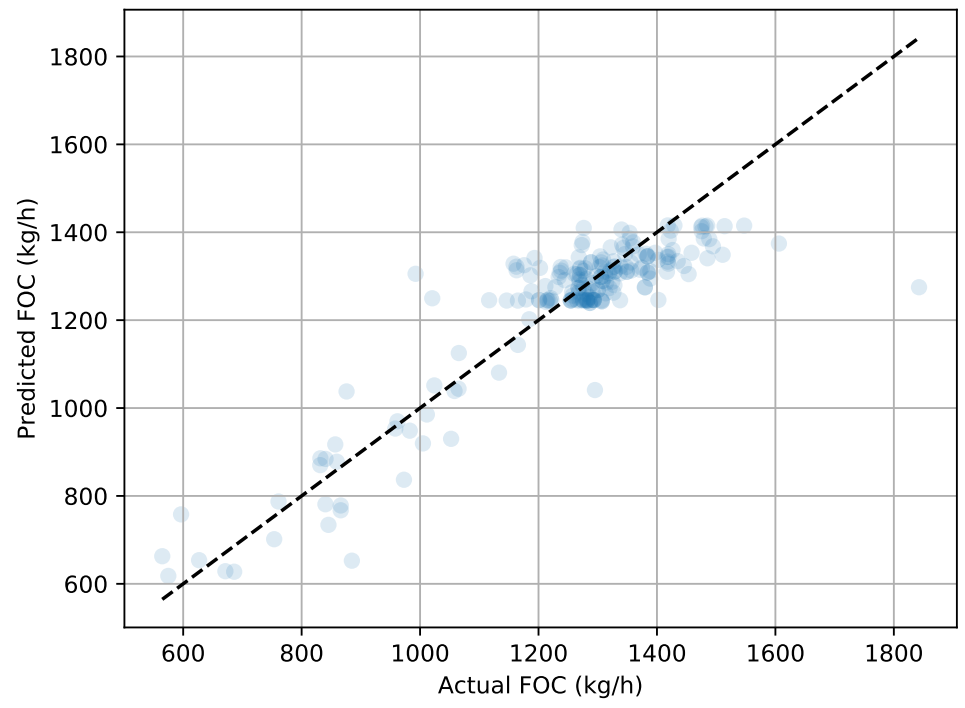

Figure 5: Comparison of actual (x-axis) vs. predicted (y-axis) FOC

\subsection{Weather routing}

Following the FOC model validation, the routing framework of Section 3.4 was applied to the route described above. The framework was applied for two case studies where the vessel is sailing at two speeds, 11 knots and 14.5 knots. The aforementioned vessel speeds refer to two typical scenarios of vessel speed whilst fully ladden, and in ballast condition. Five optimal routes were identified for each case study, presented in Figures 7 and 8 accordingly. In Figure 7 where the vessel is sailing at 11 knots, the red (thickest) line corresponds to the route that minimises $\mathrm{FOC}$ followed by the yellow, blue, green, and pink lines in diminishing order of thickness and optimality. In this case, all routes suggested follow the contour of Africa before heading through the strait of Gibraltar and then contour the Iberian peninsula till Marseille anchorage is reached. In Figure 


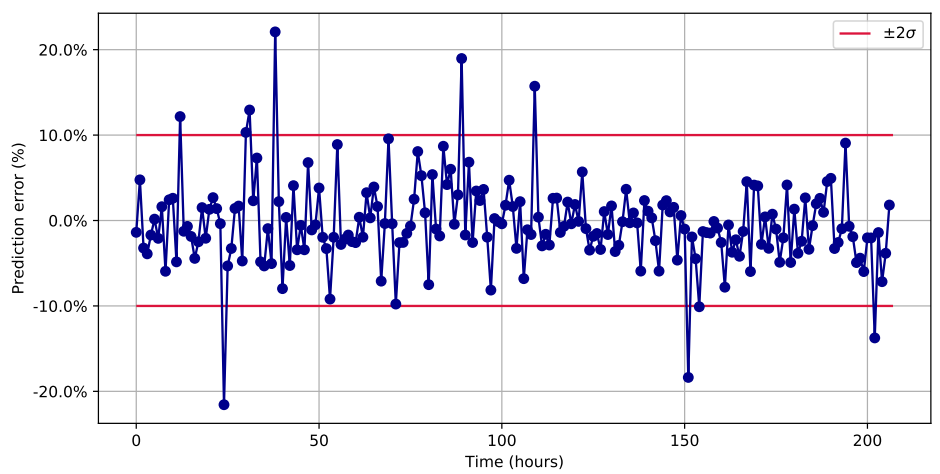

Figure 6: Error between predicted and actual FOC in time-series form.

8 where the case study vessel is sailing at 14.5 knots, similar routes are proposed with the

While routes identified in both cases are mostly similar, differences can be observed close to Mauritania where, mostly due to weather differences and the vessel's performance changing under varying speeds, a different optimal path is identified.

In cases where larger route deviations are permitted in order to avoid adverse weather conditions, such as a crossing of the Atlantic, the weather optimisation module would return paths with lower overlap levels. However such data were not available at the time of the publication of this paper.

\section{Conclusions}

This study presents a data-driven framework for decision support in the selection of optimal ship routes based on available weather forecasts and the calculation of FOC over alternative routes. The main findings of the research conducted are as follows:

- The initial process of acquiring, pre-processing and analysing a dataset containing raw sailing measurements from a ship is elaborated. 


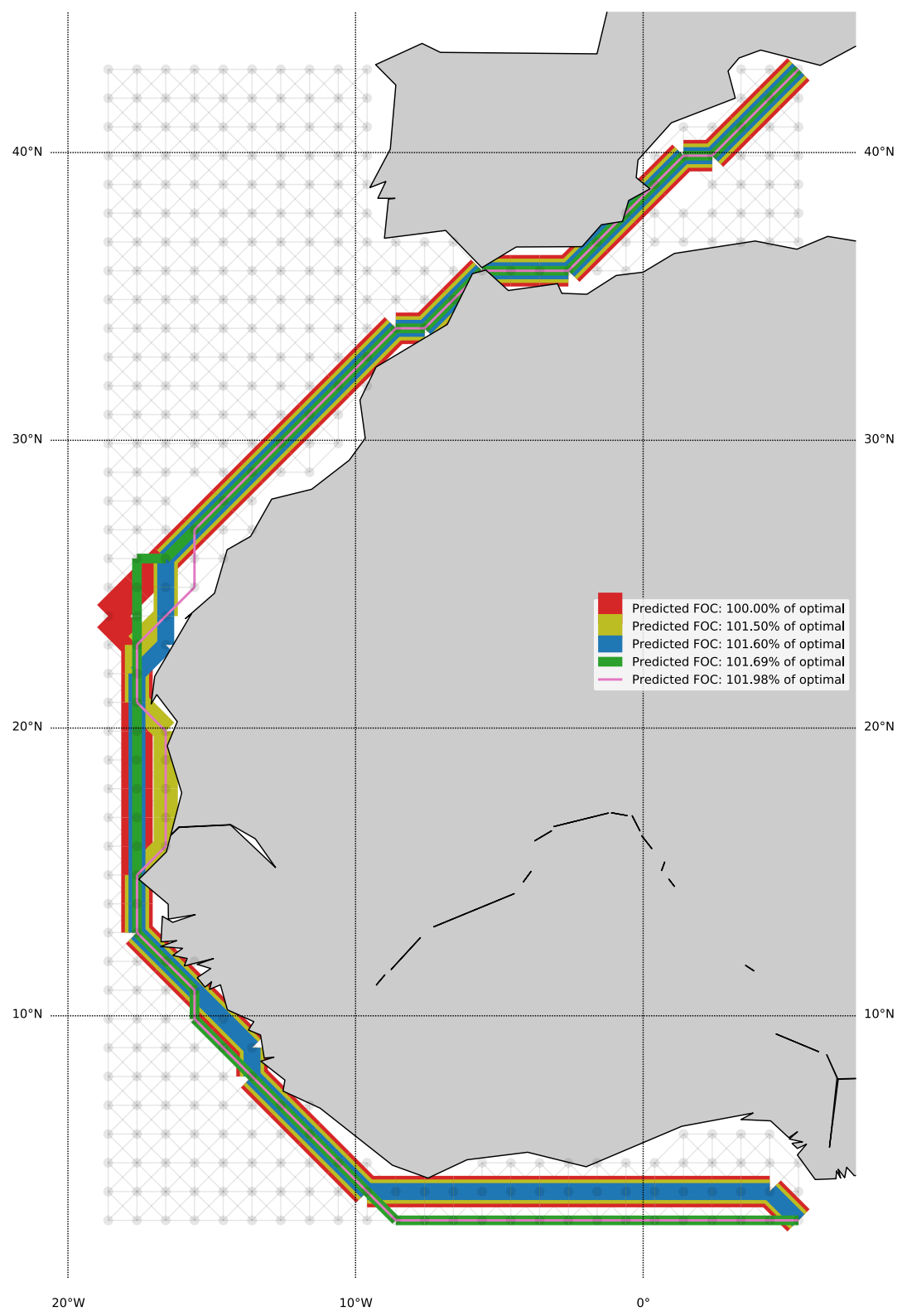

Figure 7: Optimal routes identified when vessel is sailing at 11 knots. 


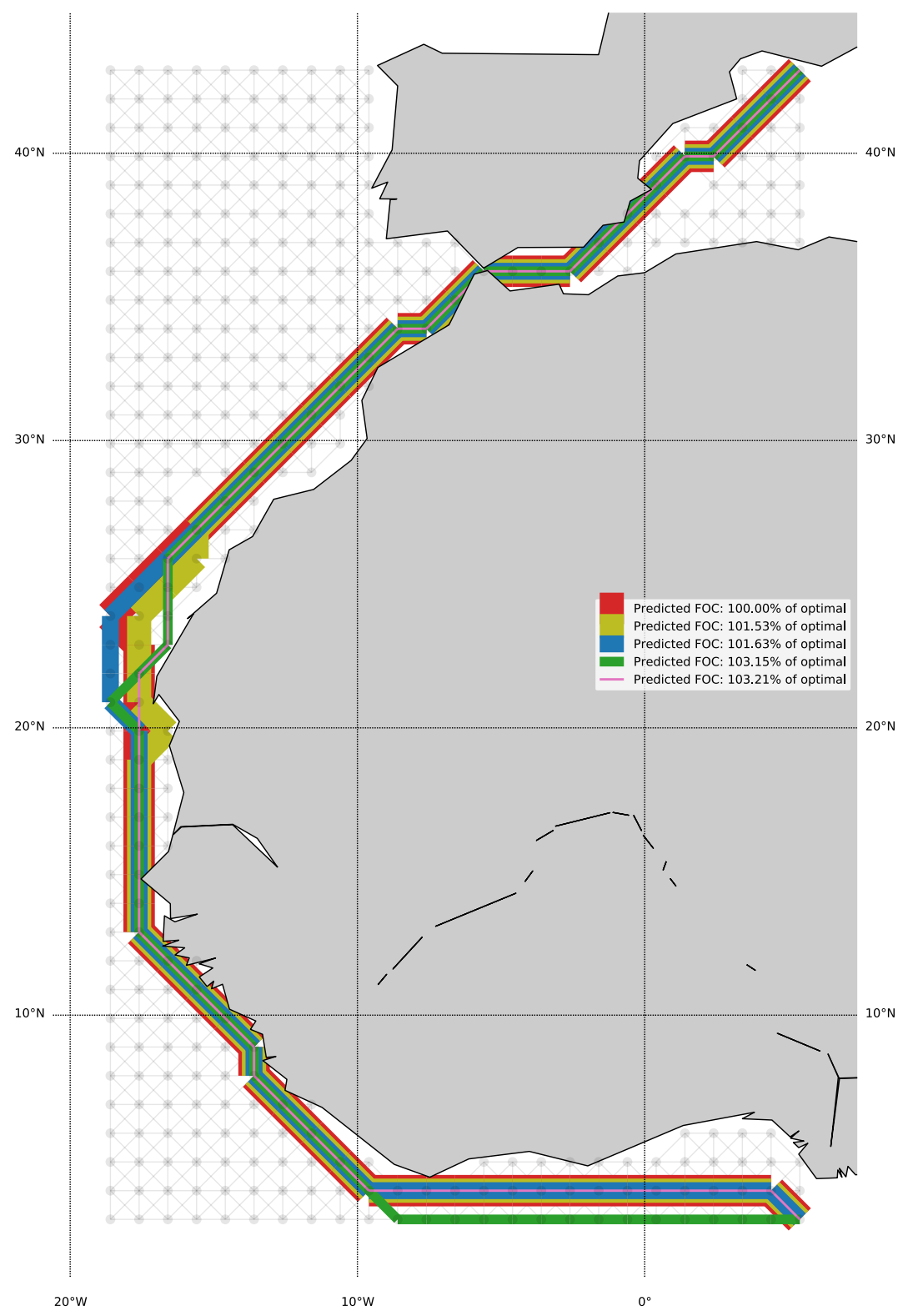

Figure 8: Optimal routes identified when vessel is sailing at 14.5 knots. 
- The process to derive a data-driven Deep Neural Network (DNN) model that can adequately represent the $\mathrm{FOC}$ of a vessel sailing under varying conditions is then included.

- Moreover, the FOC model is utilised as part of a route optimisation process that combines the data-driven aspect of the $\mathrm{FOC}$ with a modification of Dijkstra's algorithm to allow for time-dependent route optimisation.

- KPIs pertinent to ship efficiency and performance are also suggested to enhance the route optimisation process and help identify when maintenance is required.

- The above are verified and validated through a case study based on actual ship data.

The proposed methodology was elaborated and showcased through the case study of a 160,000 tonne DWT crude oil tanker sailing between the Gulf of Guinea and the Marseille anchorage. As this was a data-driven, black-box approach to weather routing, the only required data were historical vessel sailing data and a suitably spatiotemporal weather forecast without any additional domain knowledge. Therefore, the proposed framework can be applied to any route and vessel type without significant modifications. It is expected that the methodology developed in this study will be used to produce more accurate and robust vessel routes based on weather and FOC minimisation. This will help monitor and reduce vessels' $\mathrm{FOC}$ and accordingly $\mathrm{CO}_{2}$ emissions and provide the opportunity to ship owners and managers to select among a number of different ship routes when considering varying weather conditions and vessel hull and engine degradation.

\section{Acknowledgements}

a The work presented in this paper is partially funded by Integrated Ship En365 ergy \& Maintenance Management System (ISEMMS) project. ISEMMS project has received research funding from Innovate UK under Project No. 102435. This 
publication reflects only the authors' views and Innovate UK is not liable for any use that may be made of the information contained within. The authors would like to also acknowledge the assistance of the consultancy firm that provided the data utilised and verified the results presented in this paper, however they must remain anonymous for confidentiality reasons due to the sensitive data within this paper.

\section{References}

Acomi, N., Acomi, O.C., 2014. Improving the Voyage Energy Efficiency by Using EEOI. Procedia - Social and Behavioral Sciences 138,

๑ 531-536. URL: https://www.sciencedirect.com/science/article/pii/ S1877042814041548, doi $10.1016 /$ J . SBSPRO. 2014.07.234.

Armstrong, V.N., Banks, C., 2015. Integrated approach to vessel en-

\ ergy efficiency. Ocean Engineering 110, 39-48. URL: https://www.

380 sciencedirect.com/science/article/pii/S0029801815005612, doi 10. 1016/J.OCEANENG.2015.10.024

Bergstra, J.S., Bardenet, R., Bengio, Y., Kégl, B., 2011. Algorithms for

口. Hyper-Parameter Optimization. URL: http://papers.nips.cc/paper/ 4443-algorithms-for-hyper-parameter-optimizat.

385 Beşikçi, E.B., Arslan, O., Turan, O., Ölçer, A., 2016. An artificial neural network based decision support system for energy efficient ship operations. Computers \& Operations Research 66, 393

a - 401. URL: http://www.sciencedirect.com/science/article/pii/ S0305054815000842, doi/https://doi.org/10.1016/j.cor.2015.04.004.

Cheliotis, M., Gkerekos, C., Lazakis, I., Theotokatos, G., 2019. A novel data condition and performance hybrid imputation method for energy efficient operations of marine systems. Ocean Engineering 188,

口 106220. URL: https://www.sciencedirect.com/science/article/pii/ S0029801819304056, doi 10.1016/J. OCEANENG.2019.106220. 
Coraddu, A., Oneto, L., Baldi, F., Anguita, D., 2017. Vessels fuel consumption forecast and trim optimisation: A data analyt-

口 ics perspective. Ocean Engineering 130, 351 - 370. URL: http: //wWw.sciencedirect.com/science/article/pii/S0029801816305571, doi:https://doi.org/10.1016/j.oceaneng.2016.11.058.

Dijkstra, E.W., 1959. A note on two problems in connexion with graphs. Nu-

口 merische Mathematik 1, 269-271. URL: http://link.springer.com/10. 1007/BF01386390, doi $10.1007 / \mathrm{BF} 01386390$.

Gkerekos, C., Lazakis, I., Papageorgiou, S., 2018. Leveraging Big Data for Fuel Oil Consumption Modelling, in: Procedings of the 17th Conference on 405 Computer and IT Applications in the Maritime Industries (COMPIT'18), pp. $144-152$.

Gkerekos, C., Lazakis, I., Theotokatos, G., 2019. Machine learning models for predicting ship main engine Fuel Oil Consumption: A comparn ative study. Ocean Engineering 188, 106282. URL: https://www.

sciencedirect.com/science/article/pii/S0029801819304561, doi:10. 1016/J.OCEANENG.2019.106282.

Goodfellow, I., Bengio, Y., Courville, A., 2016. Deep learning. MIT Press. URL: https://mitpress.mit.edu/books/deep-learning.

Gurney, K., 1997. An introduction to neural networks. UCL Press. URL: https://dl.acm.org/citation. cfm?id=523781.

Hastie, T., Tibshirani, R., Friedman, J., 2009. The Elements of Statistical Learning. Springer Series in Statistics, Springer New York, a New York, NY. URL: https://link.springer.com/content/pdf/

1. 10.1007\%2F978-0-387-84858-7.pdf\%0Ahttp://link.springer.com/

420 10.1007/978-0-387-84858-7http://link.springer.com/10.1007/ 978-0-387-84858-7, doi $10.1007 / 978-0-387-84858-7$. 
Kuhn, M., Johnson, K., 2013. Applied Predictive Modeling. Springer

п New York, New York, NY. URL: http://link.springer.com/10.1007/ 978-1-4614-6849-3, doi $10.1007 / 978-1-4614-6849-3$.

Lin, Y.H., Fang, M.C., Yeung, R.W., 2013. The optimization of ship weatherrouting algorithm based on the composite influence of multi-dynamic el口 ements. Applied Ocean Research 43, 184-194. URL: https://www. a sciencedirect.com/science/article/pii/S0141118713000679 doi 10. 1016/J.APOR . 2013.07.010.

Lu, R., Turan, O., Boulougouris, E., Banks, C., Incecik, A., 2015. A semi-empirical ship operational performance prediction model for voyage optimization towards energy efficient ship-

口 ping. Ocean Engineering 110, Part B, 18 - 28. URL: http: //wWw.sciencedirect.com/science/article/pii/S0029801815003558, doi:https://doi.org/10.1016/j.oceaneng.2015.07.042. energy Efficient Ship Design and Operations.

MAN B\&W Diesel A/S, 2004. Instruction Book 'Operation' for 50-108MC/MCC Engines. 2 ed., Copenhagen, DK.

Petersen, J.P., Winther, O., Jacobsen, D.J., 2012. A Machine-Learning Approach to Predict Main Energy Consumption under Realistic Operational Conditions. Ship Technology Research 59, 64-72. URL: http: a //www.tandfonline.com/doi/full/10.1179/str.2012.59.1.007, doi:10. $1179 /$ str.2012.59.1.007.

Prpić-Oršić, J., Vettor, R., Faltinsen, O.M., Guedes Soares, C., 2016. The influence of route choice and operating conditions on fuel consumption and CO2 emission of ships. Journal of Marine Science and Technology 21, 434-457. URL: http://link. springer.com/10.1007/s00773-015-0367-5, doi:10.1007/s00773-015-0367-5.

Roh, M.I., 2013. Determination of an economical shipping route considering the effects of sea state for lower fuel consumption. In- 
ternational Journal of Naval Architecture and Ocean Engineering 5,

q 246-262. URL: https://www.sciencedirect.com/science/article/pii/ S2092678216303958, doi 10.2478/IJNAOE-2013-0130.

Shah, A.D., Bartlett, J.W., Carpenter, J., Nicholas, O., Hemingway, H., 2014. Comparison of Random Forest and Parametric Imputation Models for Imputing Missing Data Using MICE: A CALIBER Study. American Journal of Epidemiology 179, 764-774.

1 URL: http://www.ncbi.nlm.nih.gov/pubmed/24589914http://www.

n pubmedcentral.nih.gov/articlerender.fcgi?artid=PMC3939843https:

//academic.oup.com/aje/article-lookup/doi/10.1093/aje/kwt312, doi:10.1093/aje/kwt312

Srivastava, N., Hinton, G., Krizhevsky, A., Sutskever, I., Salakhutdinov, R., 2014. Dropout: A Simple Way to Prevent Neural Networks from Overfitting.

q Journal of Machine Learning Research 15, 1929-1958. URL: http://jmlr. org/papers/v15/srivastava14a.html

Stopford, M., 2009. Maritime Economics. Third ed., Routledge.

Tsitsilonis, K.M., Theotokatos, G., 2018. A novel systematic methodology for ship propulsion engines energy management. Journal of Cleaner Production 204, 212-236. doi:10.1016/j.jclepro.2018.08.154

470 Veneti, A., Makrygiorgos, A., Konstantopoulos, C., Pantziou, G., Vetsikas, I.A., 2017. Minimizing the fuel consumption and the risk in maritime transportation: A bi-objective weather routing approach. Computers \& Operations Research 88, 220-236. URL: https://www.sciencedirect.com/science/ article/pii/S0305054817301934, doi10.1016/J.COR.2017.07.010

475 Vettor, R., Guedes Soares, C., 2016. Development of a ship weather n routing system. Ocean Engineering 123, 1-14. URL: https://www.

a sciencedirect.com/science/article/pii/S0029801816302141, doi 10. 1016/J.OCEANENG.2016.06.035 
Walther, L., Rizvanolli, A., Wendebourg, M., Jahn, C., 2016. Modeling and

of e-Navigation and Maritime Economy 4, 31-45. URL: https://www.

a Sciencedirect.com/science/article/pii/S2405535216300043, doi 10. 1016/J.ENAVI.2016.06.004.

Wang, S., Ji, B., Zhao, J., Liu, W., Xu, T., 2018. Predicting ship fuel consumption based on LASSO regression. Transportation Research Part D: Transport 口 and Environment 65, 817-824. URL: https://www.sciencedirect.com/

a Science/article/pii/S1361920917302109, doi 10.1016/J.TRD.2017.09. 014 . 